\title{
Leveraging feedback: A systems approach to successful behaviour change in weight management
}

\section{Penny Deck}

\author{
Simon Fraser University
}

Approximately one in four Canadian adults are obese, a number that has doubled during the past 30 years. ${ }^{1}$ The increasing prevalence represents both a health and economic burden and highlights the need for novel approaches to address obesity. Over the past decade there has been a shift in the academic literature to acknowledge obesity as a complex problem. Embracing the complexity of obesity opens the door for new strategies for solving the obesity epidemic, yet few pursue these opportunities. Solving complex problems requires a systems approach. Feedback, a process that allows a system to alter its behaviour or functions in order to achieve a desired outcome, is an important defining characteristic of complex systems. Although feedback loops provide potential for developing new solution strategies, they remain a poorly understood and under-utilized leverage point for intervention. ${ }^{2}$ One area that will benefit from an improved understanding of feedback is individual health behaviour change. Feedback can be leveraged to both help assess the effectiveness of current interventions as well as to facilitate the development of new tools to assist in weight management.

A feedback loop is the basic operating unit of a system. ${ }^{3}$ A simple generic feedback loop is illustrated in Figure 1. Information about the system's state is compared with a

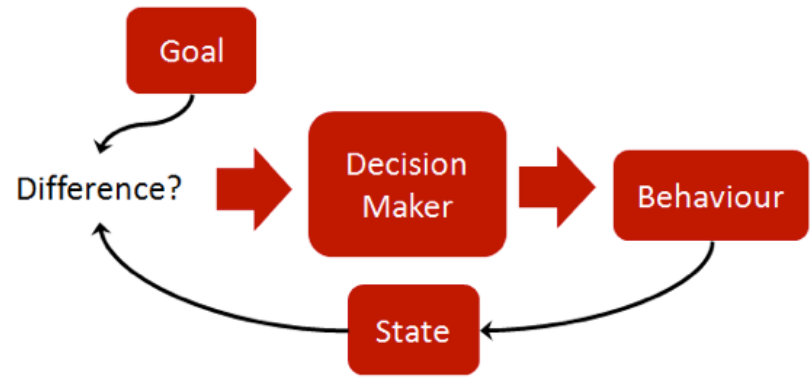

Figure 1: A Generic Feedback Loop desired goal, forming a closed chain of connections that allows for decisions to alter behaviour that will change the actual state. Feedback loops may either reduce the occurrence of a behaviour or promote more of it (Figure 2). For example, consider a sedentary individual who wishes to become more active. However, when exercising, he feels uncomfortable and worries that others are staring at him. This discomfort serves as feedback which may decrease the frequency of exercising or stop the behaviour completely. An example of a reinforcing feedback loop is the cycle of binge eating. Consider an individual who eats because she is unhappy. Eating more may cause greater unhappiness; this in turn, may lead to more overeating, again leading to even more unhappiness, a vicious and repetitive cycle. Feedback here reinforces the behaviour, enhancing an established pattern, which, in this case, potentially contributes to weight gain.

In order for interventions that leverage feedback to be effective, the feedback itself must be successful. Successful feedback elicits change within the system, is selfdetermined, and is able to evolve and adapt to changing conditions. ${ }^{4}$ Determining if feedback does produce a change in the system may be challenging. For some feedback loops, there may be a long time delay before change occurs. For example, an individual trying to lose weight by reducing energy intake typically does not see an immediate decrease in body weight. Interventions supporting individual behavioural change by creating new feedback loops may be more effective if there is a shorter time period between initiating a change in behaviour and the response that feeds back into the decision making process. Furthermore, the relevance of feedback must be self-determined in order to motivate a change in behaviour. ${ }^{4}$ Only the individual can identify what is important enough to act as a trigger. Sources of motivation vary with the individual, perhaps resulting from a life-threatening heart attack, having 


\section{Balancing Loops}

Reduce the occurrence of a

behaviour
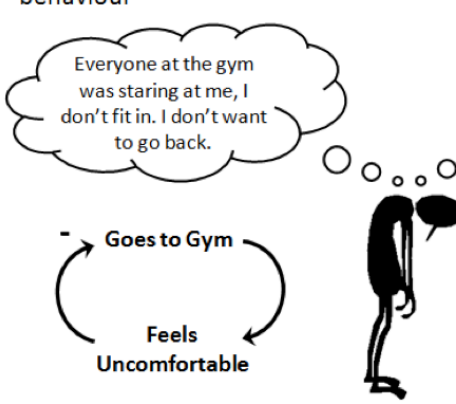

Figure 2: Balancing vs. Reinforcing Feedback Loops

children, or the break-up of a long-term relationship. ${ }^{5}$ In addition, adaptability ensures that feedback is still effective despite changes in the system. For example, parents tend to assess the healthfulness of their own child's weight based on a comparison to other children. This in turn, may affect parents' decisions to seek help for their children. Research from the UK suggests that the body weight perceived as healthy and acceptable by parents is increasing. ${ }^{6}$ This illustrates how a change in the system (e.g. increasing weight norms) requires adaptability (e.g. parents modifying their perceptions of what is healthy) in order to ensure they seek help when appropriate. Applying an understanding of these characteristics may be beneficial in ensuring the success of interventions based on feedback.

The effectiveness of feedback is also influenced by whether the feedback is based on the outcome of the behaviour or the process by which the outcome is changed. ${ }^{7}$ Outcome based feedback provides information about the end result. For an individual with the goal of losing weight, the outcome measure is body weight as it changes over time. Outcome feedback exhibits longer delays; it takes time for a change in body weight to occur. To lose weight, the individual must either change dietary intake or physical activity levels to produce a change, both of which change weight indirectly. The longer delays increase risk of oscillations ${ }^{3}$ such as that seen with weight cycling (a repeating cycle of weight loss followed by weight gain) which may lead to increased difficulty with additional weight loss attempts.

Process based feedback provides information about action, or the behaviour itself. In the weight loss example, one possible feedback strategy would be using a pedometer to track daily steps taken. As the feedback is about the behaviour itself, there are minimal time delays. Because the information is received more quickly and is specific to walking rather than weight, it is possible to modify the behaviour directly, such as making a decision to add more walking to the day's activities. Consequently, process based feedback may offer greater potential for success as it minimizes delays and allows for direct adaptation.

This understanding of feedback from a systems perspective offers a new framework for approaches to solving the obesity epidemic. Self-monitoring is an important component of behavioural weight loss strategies, yet success of such programs remains mixed, with adherence cited as a common problem. ${ }^{8}$ Applying this knowledge about feedback may help construct improved tools for self-monitoring. For example, mobile technologies that facilitate tracking of behaviours ${ }^{9}$ can be better designed to leverage feedback loops to support behaviour change at the individual level. Strategies that support self-determination by allowing individuals to identify what is most relevant to their specific situation, ${ }^{10}$ that can be modified over time and in response to changing circumstances, and that focus on process rather than outcomes, promise to improve the success of the intervention. Feedback loops represent a gap in intervention strategies ${ }^{2}$ and this framework will help identify the means to improve existing feedback loops as well as create new ones, adding an important tool to the arsenal of strategies used to combat the current obesity epidemic.

\section{Acknowledgments}

The author would like to thank Dr. Diane Finegood and the Chronic Disease Systems Modeling Lab at Simon Fraser University for their support.

\section{References}

1. Obesity in Canada: A Joint Report from the Public Health Agency of Canada and the Canadian Institute for Health Information. 2011. Available from: http://www.phac-aspc.gc.ca/hp-ps/hl-mvs/oic-oac/assets/pdf/oic-oaceng.pdf

2. Malhi L, Karanfil Ö, Merth T, Acheson M, Palmer A, Finegood DT. Places to intervene to make complex food systems more healthy, green, fair, and affordable. Journal of Hunger \& Environmental Nutrition. 2009;4(3-4):46676. Available from: http://dx.doi.org/10.1080/19320240903346448

3. Meadows DH. Leverage points - places to intervene in a system. In: Thinking in Systems. White River Junction: Chelsea Green Publishing Company; 2008.

4. Wheatley M, Kellner-Rogers M. What do we measure and why? Questions about the uses of measurement. Journal for Strategic Performance Measurement. 1999 June.

5. Ogden J, Hills L. Understanding sustained behavior change: the role of life crises and the process of reinvention. Health. 2008;12(4):419-37. Available from: http://www.ncbi.nlm.nih.gov/pubmed/18818273 
6. Carnell S, Edwards C, Croker H, Boniface D, Wardle J. Parental perceptions of overweight in 3-5 y olds. International Journal of Obesity. 2005;29(4):3535. Available from: http://www.ncbi.nlm.nih.gov/pubmed/15768040

7. Medvedeff M, Gregory JB, Levy P. How attributes of the feedback message affect subsequent feedback seeking: The interactive effects of feedback sign and type. Psychologica Belgica. 2008;48(2-3):109-25.

8. Burke LE, Wang J, Sevick MA. Self-monitoring in weight loss: a systematic review of the literature. Journal of the American Dietetic Association. 2011;111(1):92-102. Available from: http://www.ncbi.nlm.nih.gov/ pubmed/21185970
9. Rao A, Hou P, Golnik T, Flaherty J, Vu S. Evolution of data management tools for managing self-monitoring of blood glucose results: a survey of iPhone applications. Journal of Diabetes Science and Technology. 2010 Jul;4(4):949-57. Available from: http://www.ncbi.nlm.nih.gov/pmc/ articles/PMC2909529/

10. Bar-Yam Y. Making Things Work: Solving Complex Problems in a Complex World. NECSI Knowledge Press; 2005.

\section{Penny Deck}

Penny Deck is currently pursuing a PhD in the Department of Biomedical Physiology an Kinesiology in the Faculty of Science at Simon Fraser University. Her research interests include applications of complex systems to behaviour change in developing solutions to chronic disease and for health promotion. 